\title{
Anti-inflammatory and neuroprotective effects of sanguinarine following cerebral ischemia in rats
}

\author{
QIN WANG, PENG DAI, HAN BAO, PING LIANG, WEI WANG, AN XING and JIANBIN SUN \\ State Key Laboratory of Cancer Biology, Department of Pharmacogenomics, School of Pharmacy, \\ The Fourth Military Medical University, Xi'an, Shaanxi 710032, P.R. China
}

Received December 15, 2015; Accepted February 25, 2016

DOI: $10.3892 /$ etm.2016.3947

\begin{abstract}
Stroke is one of the leading causes of mortality worldwide. Protective agents that can diminish injuries caused by cerebral ischemia-reperfusion (I/R) are important in alleviating the harmful outcomes of stroke. The aim of the present study was to investigate the protective role of sanguinarine in cerebral I/R injury. A rat middle cerebral artery occlusion model was used to assess the clinical effect of sanguinarine, and inflammatory cytokines in the serum were detected by ELISA. Western blotting was performed to examine the change in levels of apoptosis-associated proteins in the injured brains. The results suggested that sanguinarine, an anti-inflammatory agent derived from the roots of Sanguinaria canadensis, improved the state of cerebral ischemia in a rat model. The data demonstrated that when rats were treated with sanguinarine prior to middle cerebral artery occlusion, the infarct volume was reduced significantly. The inflammatory factors tumor necrosis factor- $\alpha$, interleukin (IL)- 6 and IL-1 $\beta$ were measured in sanguinarine and vehicle-treated groups using an enzyme-linked immunosorbent assay, and the expression levels of the three factors were significantly reduced following treatment with sanguinarine $(\mathrm{P}<0.05)$. In addition, western blot analysis demonstrated that the ratio of $\mathrm{B}$-cell lymphoma $2 / \mathrm{Bcl}-2$-associated $\mathrm{X}$ protein was significantly increased following treatment with sanguinarine $(\mathrm{P}<0.05)$. The study demonstrated that sanguinarine exerts a protective effect in cerebral ischemia, and that this effect is associated with the anti-inflammatory and anti-apoptotic properties of sanguinarine.
\end{abstract}

Correspondence to: Dr Qin Wang, State Key Laboratory of Cancer Biology, Department of Pharmacogenomics, School of Pharmacy, The Fourth Military Medical University, 169 West Changle Road, Xi'an, Shaanxi 710032, P.R. China

E-mail: maomao_snnu@163.com

Key words: sanguinarine, cerebral ischemia, middle cerebral artery occlusion, inflammation, apoptosis

\section{Introduction}

Sanguinarine, with a formula of 13-methyl-[1,3]benzodiox olo[5,6-c]-1,3-dioxolo[4,5-i]phenanthridinium, is an active ingredient derived from the roots of Sanguinaria canadensis, the seeds of Argemone mexicana and from various other poppy-fumaria species $(1,2)$. Sanguinarine is of great interest from a practical and research perspective due to its widespread and evident biological activities (3). It is used as a naturopathic therapy for treating infections and managing pain, and as an expectorant, sedative and emetic (3). Sanguinarine has been demonstrated to exhibit anti-oxidant, anti-tumor, anti-inflammatory and anti-microbial properties $(1,4-7)$. However, the effect of sanguinarine on neuroprotection has not been widely investigated.

Stroke or cerebral ischemia is one of the leading causes of long-term disabilities and mortality worldwide. Ischemic injury is caused by a permanent or transient reduction in cerebral blood flow in the brain artery $(8,9)$. As a result of oxygen depletion, brain tissue is exposed to a dramatic decline in blood flow, $\mathrm{Ca}^{2+}$ overload, mitochondrial and DNA damage, excessive glutamate-receptor activation and oxygen radical formation $(8,9)$. Necrosis and apoptosis then occur in brain cells, leading to eventual cell death. Various mechanisms of cerebral ischemia have been identified in previous studies, including excitotoxicity, peri-infarct depolarizations, inflammation and programmed cell death $(10,11)$. Following ischemia, inflammatory cells (including microglia and blood-derived leukocytes) are activated and accumulate in the brain tissue, resulting in inflammatory injury.

During the processes of cerebral ischemia or stroke, certain molecular factors are generated, which then activate components of innate immunity, promote inflammatory signaling and cause tissue damage (12). The most extensively studied inflammatory cytokines associated with stroke include interleukin (IL)-1 $\beta$, IL-6, IL-10 and tumor necrosis factor- $\alpha$ (TNF- $\alpha$ ). These molecules gather more leukocytes to the site of ischemia, resulting in further loss of nerve cells, increased apoptosis or necrosis in cerebral tissue, and an increased area of cerebral infarction (13). All these functions are considered to induce apoptosis. Members of the B-cell lymphoma 2 (Bcl-2) family serve an important role in cerebral ischemia. In particular, Bcl-2 (which is an anti-apoptotic protein) and $\mathrm{Bcl}-2$-associated $\mathrm{X}$ protein (Bax; which is a pro-apoptotic 
protein) regulate apoptosis by mutual suppression (14). Shortly following the beginning of ischemia, dephosphorylation and translocation of Bax from the cytosol to the mitochondria, as well as dimerization of Bax with antiapoptotic proteins, occur in the brain $(15,16)$. Bax is then oligomerized and activated, eventually triggering the release of apoptotic proteins stored in the mitochondrial intermembrane space, resulting in neuronal apoptosis (17).

Considering that inflammatory changes occur during the acute phase of ischemic stroke, and that inflammation serves a central role in the disease outcome, the use of anti-inflammatory treatment agents may benefit patients suffering an ischemic stroke. Thus, the development of anti-inflammatory drugs is required for the recovery from stroke. Sanguinarine is known to be an effective, naturally active, anti-inflammatory agent. Therefore, the current report investigated the in vivo effect of sanguinarine treatment in an animal model of middle cerebral artery occlusion (MCAO). The findings provide an insight into the sanguinarine-dependent anti-inflammatory and anti-apoptotic activities in brain injury.

\section{Materials and methods}

Animals. A total of 24 male adult Sprague-Dawley rats (age, 8-10 weeks; weight, 240-260 g) were provided by the Lab Animal Center of The Fourth Military Medical University (Xi'an, China) and housed in polypropylene cages in an air-conditioned room $\left(24^{\circ} \mathrm{C}, 50 \%\right.$ humidity) with a $12-\mathrm{h}$ dark:light cycle, allowing free access to pelleted diet and water. There were three groups in total, each with 8 rats. All experiments were performed in accordance with the Guidelines for Animal Research of The Fourth Military Medical University. The study was approved by the Ethics Committee of Medical Ethics and the Human Clinical Trial Committee of Xijing Hospital (approval no. XJYYLL-2015694).

Reagents. Sanguinarine was purchased from Shaanxi Huike Botanical Development Co., Ltd (Xi'an, China). Triphenyl tetrazolium chloride (TTC) was purchased from Amresco, LLC (Solon, OH, USA) and was dissolved in phosphate buffered saline $(\mathrm{PBS})$ at $1 \%(\mathrm{~m} / \mathrm{v})$. All ELISA kits for IL-1 $\beta$ (cat no. CSB-E08055r), IL-6 (cat no. CSB-E04640r) and TNF- $\alpha$ (cat no. CSB-E11987r) were purchased from Cusabio Biotech Co., Ltd. (Wuhan, China). All antibodies were purchased from Proteintech (Wuhan, China). The enhanced chemiluminescence kit was purchased from EMD Millipore (cat no. WBKLS0100; Billerica, MA, USA).

MCAO animal model. The MCAO model was established in male Sprague-Dawley rats as previously described $(18,19)$. Rats were anesthetized using $10 \%$ chloral hydrate (Dalian Meilun Biotech Co., Ltd., Dalian, China) throughout the surgery. MCAO was maintained for $2 \mathrm{~h}$, followed by reperfusion for $24 \mathrm{~h}$ (20). Sanguinarine was suspended in $0.5 \%$ carboxymethyl cellulose (CMC)-Na (Tianguan, Guangzhou, China) and rats were administered a dose of $15 \mathrm{mg} / \mathrm{kg}$ intragastrically. For the vehicle-treated group, equal volumes of $0.5 \% \mathrm{CMC}-\mathrm{Na}$ were administered. In the vehicle-treated group, the same volume of $0.5 \%$ CMC-Na was administered
$1 \mathrm{~h}$ prior to MCAO. Rats were anesthetized with chloral hydrate and decapitated $24 \mathrm{~h}$ after MCAO.

Neurological evaluation. Modified neurological severity score (mNSS) was determined 1 day after MCAO by two independent observers. All assessments were performed in triplicate. This scoring included evaluation of the balance, sensory, reflex and motor of rats, and scores were graded on a scale of 0-18 (21). A higher score represents a more severe injury (normal $=0$; maximal deficit $=18$ ).

Measurement of infarct volume. Rats were anesthetized with $10 \%$ chloral hydrate and decapitated following reperfusion for $24 \mathrm{~h}$. Brains were rapidly and carefully removed, and sliced into 2-mm continuous slices using a metallic brain matrix (Xinruan Informatlon Technology Co. Ltd., Shanghai, China). The slices were stained with $1 \%$ TTC at $37^{\circ} \mathrm{C}$ for $15 \mathrm{~min}$ in the dark, then fixed with $4 \%$ formaldehyde in PBS. The unstained waxy area of the brain slice was defined as infarction, and the infarct volume ratio was measured and calculated as described previously (22).

Nissl stain of rat brain. The rats treated by ischemia reperfusion after MCAO and sustained for $24 \mathrm{~h}$, then anesthetized with $10 \%$ chloral hydrate, and processed by transcardial perfusion with PBS and then with $10 \%$ neutral-buffered formalin. Next, the brains were removed and immersed in $10 \%$ fresh neutral-buffered formalin for $24 \mathrm{~h}$ and embedded in paraffin. The brains were then cut into $2 \mathrm{~mm}$ thick sections, dewaxed, rehydrated with xylene and ethanol and stained with $0.5 \%(\mathrm{~m} / \mathrm{v})$ Toluidine Blue (Sigma-Aldrich, St. Louis, MO, USA). Nissl bodies were visualized with an Eclipse Ti-U Inverted Microscope System (Nikon Corporation, Tokyo, Japan) and counted using Image-Pro Plus version 6.0 (Media Cybernetics, Inc., Rockville, MD, USA).

ELISA of TNF- $\alpha, I L-6$ and $I L-1 \beta$. The injured areas of the brain tissue were removed $24 \mathrm{~h}$ after ischemia/reperfusion (I/R) injury, washed in cold PBS and placed into a homogenate tube. Appropriate volume of PBS were added into the tubes at $4{ }^{\circ} \mathrm{C}$, to allow for the tissues to be ground into $10 \%$ homogenate. The supernatant was collected following centrifugation at 4,000 $\mathrm{x} g$ for $15 \mathrm{~min}$. The protein levels of TNF- $\alpha$ (cat no. CSB-E11987r), IL-6 (cat no. CSB-E04640r) and IL-1 $\beta$ (cat no. CSB-E08055r) were determined using ELISA kits (Cusabio Biotech Co., Ltd.) according to the manufacturer's instructions, which included the addition of $100 \mu \mathrm{l}$ of appropriately diluted samples to each well. Standards (triplicates) and blanks were run with each plate to ensure accuracy

Western blotting assay. The injured area of the brain tissue was homogenized in cold radioimmunoprecipitation assay buffer (Sigma-Aldrich). The concentrations of whole cell extracts were determined by bicinchoninic acid assay (Thermo Fisher Scientific, Inc., Waltham, MA, USA) and $20 \mu \mathrm{g}$ total proteins were loaded into each well. Protein extracts were subjected to electrophoresis on a $10 \%$ Bis-Tris protein gel (Invitrogen; Thermo Fisher Scientific, Inc., Waltham, MA, USA). The membranes were incubated with the following primary 
antibodies: Rabbit anti-Bax polyclonal antibody (1:500; cat no. 50599-2-Ig), rabbit anti-Bcl-2 polyclonal antibody (1:500; cat no. 12789-1-AP), mouse anti-GAPDH monoclonal antibody $(1: 1,000$; cat no. 60004-1-Ig). The horseradish peroxidase-conjugated goat anti-mouse/rabbit IgG were used to detect the primary antibodies. Subsequently, enhanced chemiluminescence reagent was added. The respective densities of western blots were analyzed using Quantity One 1-D Analysis Software (version 4.4; Bio-Rad Laboratories, Inc., Hercules, CA, USA).

Statistical analysis. Data are expressed as the mean \pm standard error. Multiple group comparisons were performed using one-way analysis of variance followed by Dunnett's test in order to detect inter-group differences. The difference between the mean values of two groups was assessed using a non-paired Student's t-test. SPSS version 12 (SPSS, Inc., Chicago, IL, USA) and GraphPad Prism (GraphPad Software, Inc., La Jolla, CA, USA) were used to perform all statistical analyses. All experiments were independently repeated three times. $\mathrm{P}<0.05$ were considered to indicate a statistically significant difference.

\section{Results}

Sanguinarine reduced infarct injury in $M C A O$ rats. Fig. 1 presents representative brain slices stained with TTC $24 \mathrm{~h}$ after reperfusion in vehicle- and sanguinarine-treated rats. Infarct volumes were expressed as a percentage of the intact contralateral hemisphere, and were found to be significantly lower in sanguinarine-treated rats (15.03\%), when compared with the infarct volumes of vehicle-treated animals $(38.76 \% ; \mathrm{P}<0.05)$ in the transient ischemic model of stroke.

Nissl staining was also investigated in brain samples from the three groups. The number of Nissl bodies was significantly reduced following MCAO (vehicle group), when compared with the sham group $(189.33 \pm 29.41$ vs. $506.00 \pm 30.83$, respectively; $\mathrm{P}<0.01$; Fig. 2 ). Treatment with sanguinarine significantly increased the number of Nissl bodies (328.00 \pm 45.77$)$ when compared with that in the vehicle-treated rats $(189.33 \pm 29.41 ; \mathrm{P}<0.05)$.

Sanguinarine improves functional outcome following MCAO. Prior to MCAO, the neurologic scores were normal in all animals (score $=0$ in all groups). High-grade behavioral deficits (scores $>12$ ) were recorded in all animals $60 \mathrm{~min}$ after MCAO (Fig. 3). Vehicle-treated animals exhibited severe behavioral impairments throughout the survival period including circling to the right, and the inability to balance and walk successfully on the beam. Treatment with sanguinarine significantly improved the neurologic score in comparison with the vehicle-treated rats $24 \mathrm{~h}$ after reperfusion $(\mathrm{P}<0.05$; Fig. 3).

Sanguinarine suppresses the inflammation caused by global cerebral $I / R$. ELISA was performed to detect the contents of TNF- $\alpha$, IL- 6 and IL- $1 \beta$ in injured brain tissue. Compared with brain tissues obtained from sham-operated animals, global cerebral I/R brains had a significant higher expression level of TNF- $\alpha$, IL-6 and IL-1 $\beta$ proteins (Fig. 4). Pre-treatment with sanguinarine significantly attenuated the increase in the TNF- $\alpha$, IL- 6 and IL-1 $\beta$ protein expression levels following global cerebral I/R (Fig. 4).

Sanguinarine reduces the activation of apoptotic signaling caused by global cerebral $I / R$. To investigate apoptotic signaling, the expression of the anti-apoptotic protein Bcl-2 and the pro-apoptotic protein Bax was investigated. The ratio of Bcl-2/Bax is presented in Fig. 5. Global cerebral I/R injury resulted in a significant reduction in $\mathrm{Bcl}-2$ protein expression in comparison with the sham-operated group. In addition, treatment of sanguinarine significantly increased the reduced $\mathrm{Bcl}-2$ expression $(\mathrm{P}<0.05)$. By contrast, Bax protein expression following global cerebral I/R was significantly increased in comparison with sham-operated animals. This increase in Bax protein expression was significantly ameliorated by pre-treatment with sanguinarine.

\section{Discussion}

Inflammation is a pathological marker of ischemic stroke and results in cerebric lesion; however, the mechanisms underlying this process are poorly understood (23). The central nervous and immune system interact in complex ways. The destruction of neurons induced by cerebral ischemia results in an immune response that is necessary to remove cell debris and initiate the regenerative process; however, this inflammatory response can aggravate cerebral damage and cause secondary cerebral injuries $(24,25)$. Circulating inflammatory mediators can activate the cerebrovascular endothelium and glial cells in the brain and affect ischemic brain injury (26). Cytokines are important inflammatory mediators $(27,28)$ and their role has been well established in previous in vivo and in vitro investigations. Ischemic injury results in an increase in the release of cytokines, which are involved in the necrosis of neuronal cells $(27,28)$. Among the large number of cytokines, TNF- $\alpha$, IL-1 $\beta$ and IL- 6 modulate tissue injury in stroke, and are therefore potential targets in developing treatments for stroke (29). The effect of cytokines on the extent of infarction depends on their viability in ischemic penumbra during the early phase of stoke (28). Numerous neuroprotective agents have been found to be effective in animal models of stroke; however, few studies have translated these findings into clinical applications. One reason for this may be the failure to consider clinical co-morbidities and risk factors in experimental animal models (27).

As an efficient anti-inflammatory agent, sanguinarine has been used for centuries as a therapeutic agent for the treatment of inflammatory diseases (1); however, the protective effect of sanguinarine on cerebral ischemia is poorly reported. In the present study, the effect of sanguinarine on MCAO rats was examined, and its therapeutic action was investigated in a well-controlled animal model of MCAO. The results demonstrated that pre-treatment with sanguinarine prevents delayed neuronal death in the MCAO model. In addition, it was observed that pre-treatment with sanguinarine was neuroprotective in transient cerebral ischemia in rats, as the infarct volume of MCAO rats was found to be significantly reduced in the sanguinarine-treated group. The injury of 
A

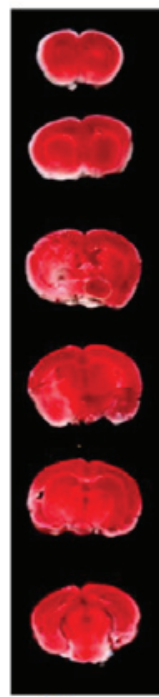

Sham

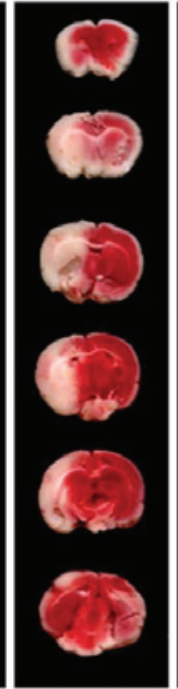

Vehicle

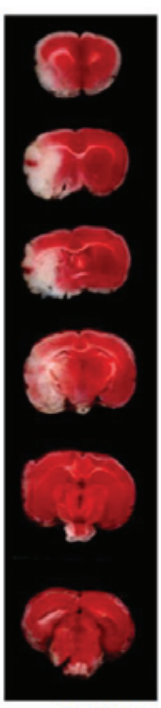

SANG

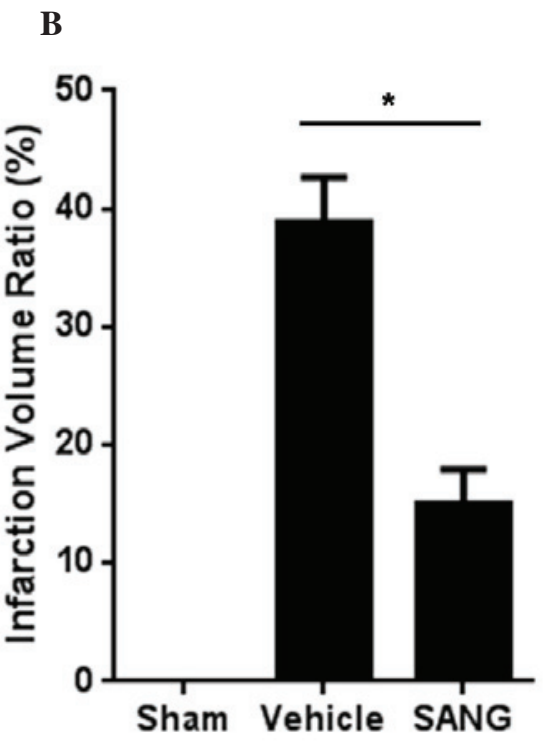

Figure 1. (A) TTC staining of the brains of rats from each group after $24 \mathrm{~h}$ of reperfusion. (B) Quantification of TTC staining. The infraction volume decreased significantly when rats were administrated SANG $(15 \mathrm{mg} / \mathrm{kg})$ prior to middle cerebral artery occlusion. Data are presented as the mean \pm standard error, $\mathrm{n}=8$. ${ }^{*} \mathrm{P}<0.05$ vs. the vehicle group. TTC, triphenyl tetrazolium chloride; SANG, sanguinarine.
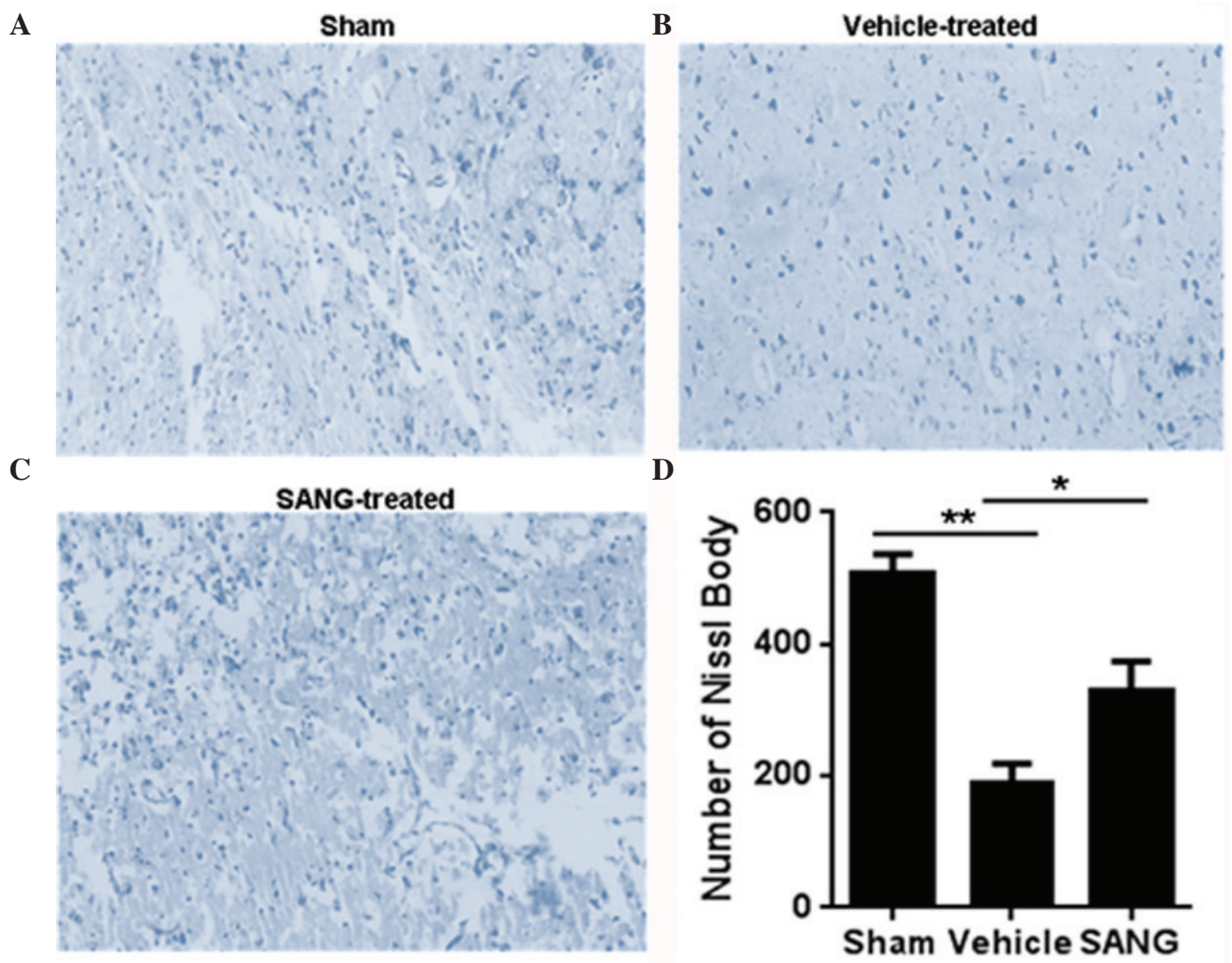

Figure 2. Nissl staining of the (A) sham (B) vehicle-treated and (C) SANG-treated groups (magnification, x100). (D) Quantification of Nissl-positive cells in the cortex. Treatment with SANG significantly increased the numbers of Nissl bodies in comparison with the vehicle-treated rats. Values are presented as the mean \pm standard error, $n=8$. ${ }^{*} \mathrm{P}<0.05$ and ${ }^{* *} \mathrm{P}<0.001$ vs. the vehicle group. SANG, sanguinarine.

brain structure was also declined in the sanguinarine-treated group.

The mechanism of action of sanguinarine was also investigated in the present study. The results demonstrated that treatment with $15 \mathrm{mg} / \mathrm{kg}$ sanguinarine resulted in a significant reduction in cerebral infarction in $\mathrm{MCAO}$-induced rats with permanent focal cerebral ischemia. In addition, sanguinarine exerted neuroprotective effects against histological injury and neurological deficits following ischemia. The expression of Bcl-2 increased, while that of Bax was reduced, and the 


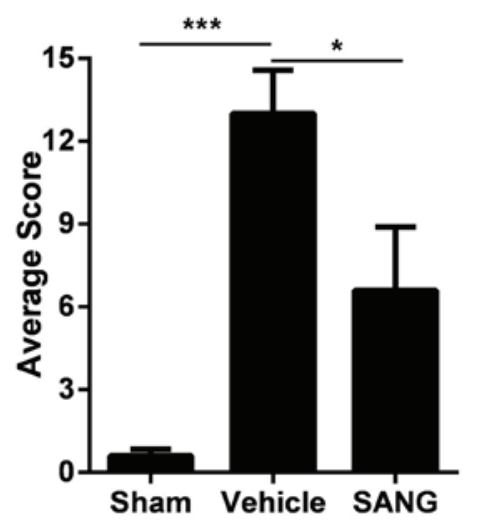

Figure 3. Mean modified neurological severity scores in rats 60 min after MCAO (healthy score $=0$; maximal injury score $=18$ ). In the SANG treated group, the average score was significantly reduced in comparison with the vehicle group. Values are presented as the mean \pm standard error, $n=8$. ${ }^{*} \mathrm{P}<0.05,{ }^{* * * *} \mathrm{P}<0.001$ vs. vehicle group. SANG, sanguinarine.

A

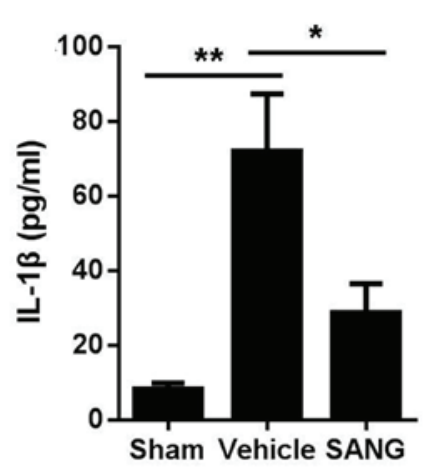

B

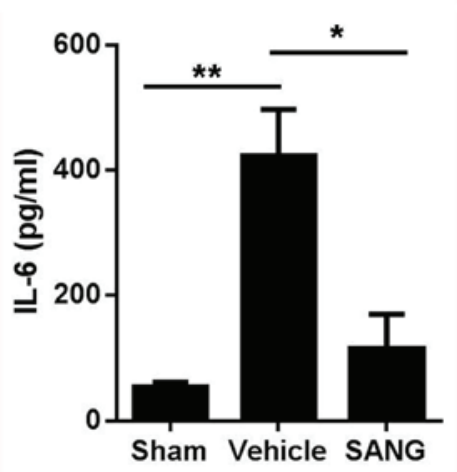

C

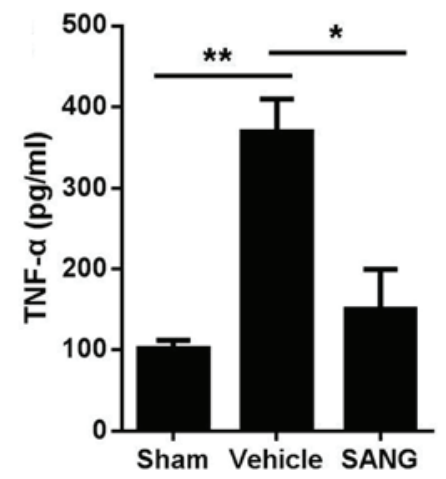

Figure 4. Effect of SANG treatment on the secretion of (A) IL-1 $\beta$, (B) IL-6 and (C) TNF- $\alpha$ in rats following middle cerebral artery occlusion rats. Data are presented as the mean \pm standard error $(\mathrm{n}=6) .{ }^{*} \mathrm{P}<0.05$ and ${ }^{* *} \mathrm{P}<0.01$ vs. vehicle group. SANG, sanguinarine; IL, interleukin; TNF- $\alpha$, tumor necrosis factor- $\alpha$.
A

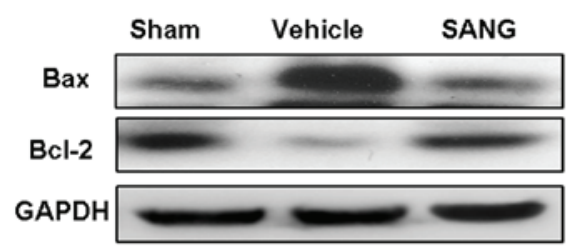

B

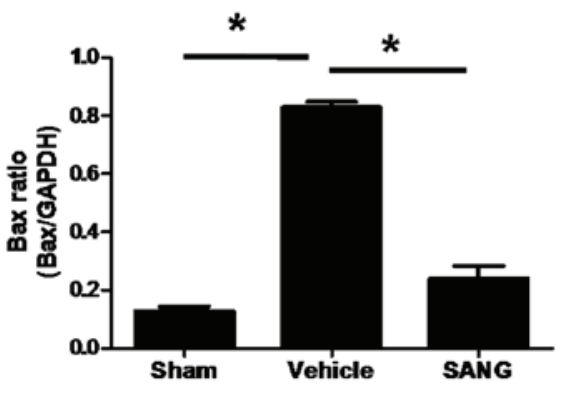

C

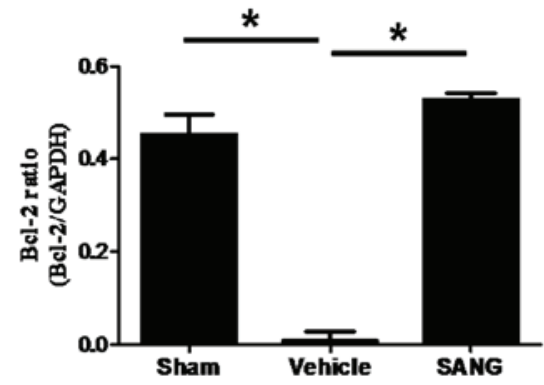

D

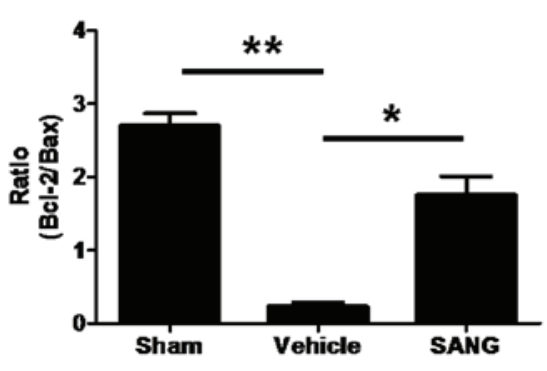

Figure 5. Effect of SANG pre-treatment on Bcl-2 and Bax protein expression in the brain. (A) Western blot bands of Bcl-2, Bax and GAPDH in sham, vehicle-treated and SANG-treated rats. Quantification of protein expression of (B) Bax/GAPDH ratio, (C) Bcl-2/GAPDH ratio and (D) the ratio of $\mathrm{Bcl}-2 / \mathrm{Bax}$. Data are presented as the mean \pm standard error, $\mathrm{n}=8 .{ }^{*} \mathrm{P}<0.05$, ${ }^{* *} \mathrm{P}<0.01$ vs. vehicle group. Bcl-2, B-cell lymphoma 2; Bax, Bcl-2-associated X protein; SANG, sanguinarine.

release of inflammatory cytokines TNF- $\alpha$, IL-1 $\beta$ and IL- 6 was reduced following pre-treatment with sanguinarine.

In conclusion, the present study demonstrated that sanguinarine treatment mediated a significant protective effect in vivo. The results suggested that sanguinarine effectively reduced apoptosis in brain cells following sanguinarine treatment, since the pro-apoptotic Bax was downregulated and the anti-apoptotic Bcl-2 was upregulated. The current study contributes towards the understanding of the protective properties of sanguinarine in MCAO animal models and the molecular mechanisms underlying this activity. Thus, to the 
best of our knowledge, this study provided the first scientific support for the use of sanguinarine for the treatment of human cerebral ischemia disease.

\section{References}

1. Niu X, Fan T, Li W, Xing W and Huang H: The anti-inflammatory effects of sanguinarine and its modulation of inflammatory mediators from peritoneal macrophages. Eur J Pharmacol 689: 262-269, 2012

2. Li H, Zhai Z, Liu G, Tang T, Lin Z, Zheng M, Qin A and Dai K: Sanguinarine inhibits osteoclast formation and bone resorption via suppressing RANKL-induced activation of NF-kappaB and ERK signaling pathways. Biochem Biophys Res Commun 430 : 951-956, 2013.

3. Mitscher LA, Drake S, Gollapudi SR and Okwute SK: A modern look at folkloric use of anti-infective agents. J Nat Prod 50: 1025-1040, 1987.

4. Obiang-Obounou BW, Kang OH, Choi JG, Keum JH, Kim SB, Mun SH, Shin DW, Kim KW, Park CB, Kim YG, et al: The mechanism of action of sanguinarine against methicillin-resistant staphylococcus aureus. J Toxicol Sci 36: 277-283, 2011.

5. Lee JS, Jung WK, Jeong MH, Yoon TR and Kim HK: Sanguinarine induces apoptosis of HT-29 human colon cancer cells via the regulation of $\mathrm{Bax} / \mathrm{Bcl}-2$ ratio and caspase-9-dependent pathway. Int J Toxicol 31: 70-77, 2012.

6. Sun M, Lou W, Chun JY, Cho DS, Nadiminty N, Evans CP, Chen J, Yue J, Zhou Q and Gao AC: Sanguinarine suppresses prostate tumor growth and inhibits survivin expression. Genes Cancer 1: 283-292, 2010.

7. Kim S, Lee TJ, Leem J, Choi KS, Park JW and Kwon TK: Sanguinarine-induced apoptosis: Generation of ROS, down-regulation of Bcl-2, c-FLIP and synergy with TRAIL. J Cell Biochem 104: 895-907, 2008.

8. Chen Y, Zhang L, Ni J, Wang X, Cheng J, Li Y, Zhen X, Cao T and Jia J: LLDT-8 protects against cerebral ischemia/reperfusion injury by suppressing post-stroke inflammation. J Pharmacol Sci 131: 131-137, 2016.

9. Shim $\mathrm{R}$ and Wong CHY: Ischemia, Immunosuppression and Infection--Tackling the Predicaments of Post-Stroke Complications. Int J Mol Sci 17: 64, 2016.

10. Broughton BR, Reutens DC and Sobey CG: Apoptotic mechanisms after cerebral ischemia. Stroke 40: e331-e339, 2009.

11. Dirnagl U, Iadecola C and Moskowitz MA: Pathobiology of ischaemic stroke: An integrated view. Trends Neurosci 22: 391-397, 1999.

12. Murakami A, Nakamura Y, Tanaka T, Kawabata K, Takahashi D, Koshimizu K and Ohigashi H: Suppression by citrus auraptene of phorbol ester-and endotoxin-induced inflammatory responses: Role of attenuation of leukocyte activation. Carcinogenesis 21: 1843-1850, 2000.

13. Farhoudi M, Najafi-Nesheli M, Hashemilar M, Mahmoodpoor A, Sharifipour E, Baradaran B, Taheraghdam A, Savadi-Oskouei D, Sadeghi-Bazargani H, Sadeghi-Hokmabadi E, et al: Effect of IMOD ${ }^{\mathrm{TM}}$ on the inflammatory process after acute ischemic stroke: A randomized clinical trial. Daru 21: 26, 2013.

14. Niizuma K, Yoshioka H, Chen H, Kim GS, Jung JE, Katsu M, Okami N and Chan PH: Mitochondrial and apoptotic neuronal death signaling pathways in cerebral ischemia. Biochim Biophys Acta 1802: 92-99, 2010.

15. Saito A, Hayashi T, Okuno S, Ferrand-Drake M and Chan PH: Overexpression of copper/zinc superoxide dismutase in transgenic mice protects against neuronal cell death after transient focal ischemia by blocking activation of the bad cell death signaling pathway. J Neurosci 23: 1710-1718, 2003.
16. Kirkland RA, Windelborn JA, Kasprzak JM and Franklin JL: A bax-induced pro-oxidant state is critical for cytochrome c release during programmed neuronal death. J Neurosci 22: 6480-6490, 2002.

17. Fiskum G, Murphy AN and Beal MF: Mitochondria in neurodegeneration: Acute ischemia and chronic neurodegenerative diseases. J Cereb Blood Flow Metab 19: 351-369, 1999.

18. Satoh T, Kosaka K, Itoh K, Kobayashi A, Yamamoto M, Shimojo Y, Kitajima C, Cui J, Kamins J, Okamoto S, et al: Carnosic acid, a catechol-type electrophilic compound, protects neurons both in vitro and in vivo through activation of the Keap1/Nrf2 pathway via S-alkylation of targeted cysteines on Keap1. J Neurochem 104: 1116-1131, 2008.

19. Wang N, Wu L, Cao Y, Wang Y and Zhang Y: The protective activity of imperatorin in cultured neural cells exposed to hypoxia re-oxygenation injury via anti-apoptosis. Fitoterapia 90: 38-43, 2013.

20. Hwang SY, Shin JH, Hwang JS, Kim SY, Shin JA, Oh ES, Oh S, Kim JB, Lee JK and Han IO: Glucosamine exerts a neuroprotective effect via suppression of inflammation in rat brain ischemia/reperfusion injury. Glia 58: 1881-1892, 2010.

21. Wang C, Pei A, Chen J, Yu H, Sun ML, Liu CF and Xu X: A natural coumarin derivative esculetin offers neuroprotection on cerebral ischemia/reperfusion injury in mice. J Neurochem 121: 1007-1013, 2012.

22. Denes A, Ferenczi S and Kovács KJ: Systemic inflammatory challenges compromise survival after experimental stroke via augmenting brain inflammation, blood- brain barrier damage and brain oedema independently of infarct size. J Neuroinflammation 8: 164, 2011.

23. Kamel $\mathrm{H}$ and Iadecola C: Brain-immune interactions and ischemic stroke: Clinical implications. Arch Neurol 69: 576-581, 2012.

24. Brea D, Sobrino T, Ramos-Cabrer P and Castillo J: Inflammatory and neuroimmunomodulatory changes in acute cerebral ischemia. Cerebrovasc Dis 27 (Suppl 1): S48-S64, 2009.

25. Benakis C, Vaslin A, Pasquali C and Hirt L: Neuroprotection by inhibiting the c-Jun N-terminal kinase pathway after cerebral ischemia occurs independently of interleukin- 6 and keratinocyte-derived chemokine (KC/CXCL1) secretion. J Neuroinflammation 9: 76, 2012.

26. Hamishehkar H, Beigmohammadi MT, Abdollahi M, Ahmadi A, Mahmoodpour A, Mirjalili MR, Abrishami R, Khoshayand MR, Eslami K, Kanani M, et al: Identification of enhanced cytokine generation following sepsis. Dream of magic bullet for mortality prediction and therapeutic evaluation. Daru 18: 155-162, 2010.

27. Lambertsen KL, Biber $K$ and Finsen B: Inflammatory cytokines in experimental and human stroke. J Cereb Blood Flow Metab 32: 1677-1698, 2012.

28. Pradillo JM, Denes A, Greenhalgh AD, Boutin H, Drake C, McColl BW, Barton E, Proctor SD, Russell JC, Rothwell NJ and Allan SM: Delayed administration of interleukin-1 receptor antagonist reduces ischemic brain damage and inflammation in comorbid rats. J Cereb Blood Flow Metab 32: 1810-1819, 2012.

29. Xu Y, Wang JY,Song XH, Qu LD, Wei RL, He FP, Wang K, Luo BY: RIP3 induces ischemic neuronal DNA degradation and programmed necrosis in rat via AIF.Scientific Reports 6: 29362, 2016. 